\title{
A novel all-optical routing architecture for optical packet switched networks
}

\author{
Bora Mocan · Mehmet Salih Dinleyici
}

Received: 25 October 2004 / Revised: 9 June 2005 / Accepted: 14 June 2005

(C) Springer Science + Business Media, Inc. 2006

\begin{abstract}
Increasing bandwidth demand, mostly driven by the Internet Protocol (IP), has made researchers consider to deploy all-optical devices into packet switched networks. Despite huge bandwidth of the optical communication links (optical fiber) the usable capacity is limited due to bottlenecks (congestions) at the switching nodes. In this paper, a novel all-optical routing architecture is proposed for optical packet switched networks. In the design, practical optical devices (gratings, threshold elements, optical delays, and couplers) have been improved and exploited in order to integrate into an alloptical routing device. The system has been implemented and simulated by using an photonics simulation package (VPI-Virtual Photonics). The packets conveying a three-bit routing information tag at the bit rate of $10 \mathrm{Gbps}$ have been successfully routed between two links. Some of the components are standard tools of the simulation package and some needed to be designed using the transfer function or theory developed in the literature. Noise and losses associated to the nonideal nature of the components are considered in the simulation as well.
\end{abstract}

Keywords Optical networks - All-optical routing · All-optical switching $\cdot$ All-optical signal processing

\section{Introduction}

Ever increasing demands of today's high speed communication networks has promoted to employ photonic technol-

\author{
B. Mocan ( $\square)$ \\ Department of Electrical and Electronics Engineering, \\ İmir Institute of Technology, Gülbahçe, \\ Urla, TR35430 İmir, Turkey \\ e-mail: boramocan@iyte.edu.tr \\ M. S. Dinleyici \\ e-mail: salihdinleyici@iyte.edu.tr
}

ogies either as optimization of existing infrastructure or as proposal of designing a novel all optical networks. In the optimization approach, the physical layer of the Open System Interconnection (OSI) protocol stack is the one that photonics technology may be applied to overcome bottlenecks of routing and switching problems of ultra-fast communication networks. A protocol transparent all-optical routing may allow straightforward extension of the existing networks into the optical regime, without any major modification of the higher layers of OSI.

In this work, a novel all-optical routing architecture based on available optical techniques and devices, is proposed. The design relies upon self-routing of the optical data packets using the packet's address information and the routing table of the network topology. Network protocols with smaller and simpler routing tables have high potential to accommodate this design. Especially latest, popular network protocols such as Multi Protocol Label Switching (MPLS) and Internet Protocol version six (IPv6) data packets might be very efficiently routed by this technique.

IPv6 has much larger address space (128 bits) than IPv4 (32 bits), which enables the use of multiple levels of hierarchy inside the address space. Each level helps to aggregate its IP space and enhance the allocation function. Service providers and organizations may have a tiered hierarchy [1]. It means there are fewer routes to analyze, fewer fields to process, and fewer decisions to make in forwarding a data packet [2]. MPLS is another forwarding technique in autonomous networks that may accommodate this approach for optimizing the routing process [3]. Global network reachability is handled at the edge and packet forwarding rules are propagated to the core network. Label Edge Routers (LER) work as interface between the MPLS network and IP Internet and assign simple labels for the incoming packet streams. A MPLS network consists of Label Switch Routers (LSR) in the core of 
the network. Within the MPLS network, traffic is forwarded via LSRs just using the labels. MPLS and IPv6 are designed to improve the routing and switching bottlenecks of the current Internet Protocol (IPv4) [3]. Therefore, the novel routing architecture proposed here might be adapted for both IPv6 and MPLS networks.

Optical buffers, optical switches, header recognition, and route decision units are key building blocks of a general optical routing node. Truly photonic routers require photonic implementations of all these functions. The lack of all-optical processors and random access memories force designers to implement specific signal processing algorithms in separate optical modules. However, the interactions among these models should be carefully considered to overcome the synchronization and stability problems that may arise on ultra-fast data rates. The use of fixed length packets can, however, significantly simplify the implementation of packet content resolution and buffering as well as packet synchronization $[4,5]$.

\section{Operation principle}

The proposed all-optical routing architecture consists of a number of optical signal processing blocks as shown in Fig. 1. At the input, only a small part of the incoming signal's energy is taken by a Y-coupler for processing to generate the routing information. The first step of the operation is header recognition and serial to parallel conversion of the header bits. Various techniques might be applied for these operations [6-8]. Every serial-to-parallel converted bit is sent to the pulse extension module, which extends the pulses in time up to the duration of the whole addresses in the routing table. Pulse extension operation makes each address bit available for the exclusive-or (XOR) operation between the entire pulse train of the routing table. The length of the routing table strongly affects the duration of the algorithm, and this limitation may be overcome by increasing the generation speed of the routing table. The routing table pulse train (RTPT) might be generated by an ultra-fast pulse generator, which is driven by an ordinary CPU. The use of an electronic CPU may not cause a delay in routing, because it is only used to keep the routing tables updated, which is a very slow process (30-120 s) compared to the packet bit rates (Gbps). RTPT, which includes "Route A" addresses, and its logical inverse $\mathrm{RTPT}^{-1}$ are required for the special all-optical XOR algorithm. If an address header carries the logic " 1 " information, the result of the XOR operation between RTPT and address bit is the RTPT itself, and if the address header has the logic " 0 " information the XOR operation results in $\mathrm{RTPT}^{-1}$. As a result, only a switching operation between RTPT and $\mathrm{RTPT}^{-1}$ that depends on the value of the address bits is sufficient to achieve an optical XOR operation. Using this useful trick an all-optical XOR is realized, which uses a counter propagating semiconductor optical amplifier assisted MachZehnder interferometer (SOA-MZI) switch [9]. In this case, the extended address bits are used as control signals while RTPT and $\mathrm{RTPT}^{-1}$ are input signals. When the routing table contains the address of the incoming packet a series of optical 1 's are produced consecutively at the XOR outputs. The XOR outputs are combined by synchronization delays and pulse combiners and passed through an suitable threshold element to generate the routing control bit that triggers the optical switch at the output port. According to the state of the routing control bit, the data packet is switched to either "Route A" or "Route B" as default. During the routing decision time interval the original data packet is kept in the optical buffer, which may be designed of fixed length fiberoptic cable, because of static delay line requirement.

All-optical routing of 12-bit fixed sized packets having 3-bit address headers is simulated using the novel routing

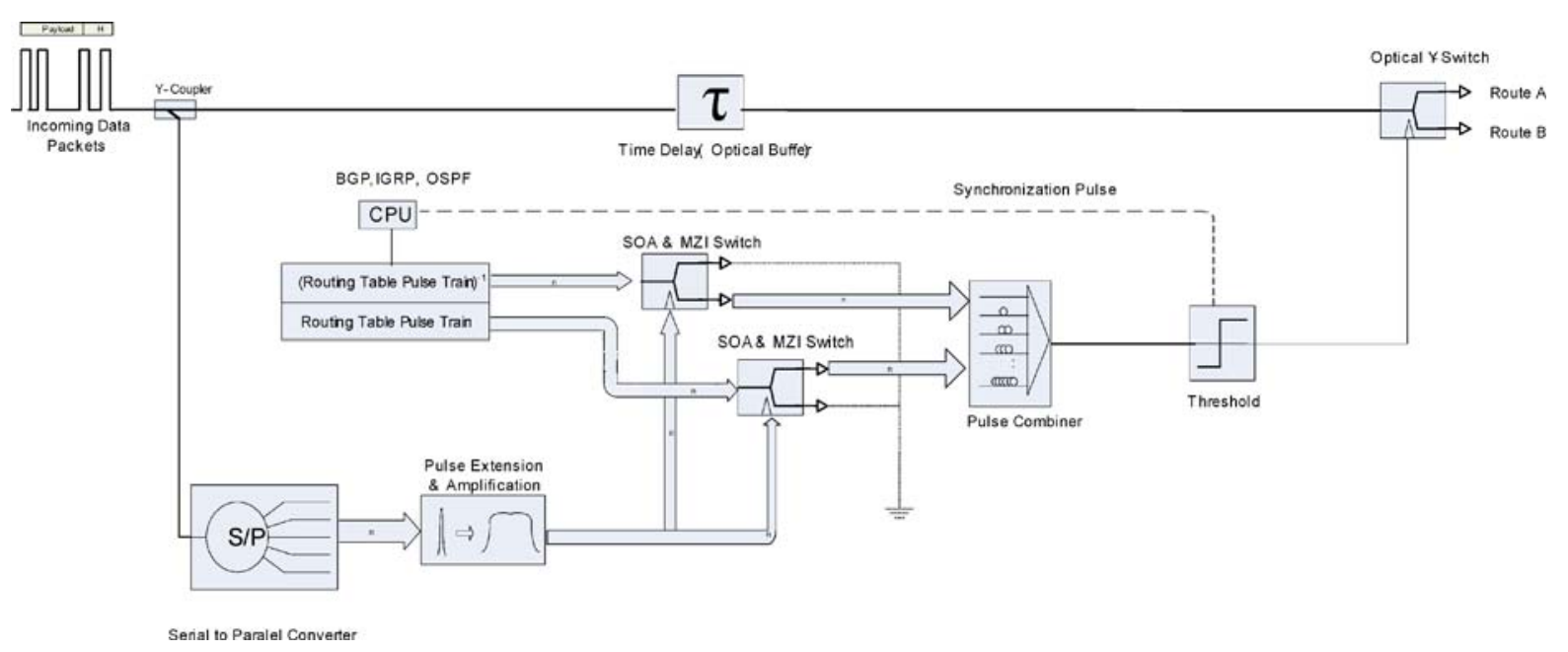

Fig. 1 Novel all-optical router architecture 
technique proposed here. VPIphotonics's VPItransmission Maker 3.1 simulation software is used as the simulation environment. It delivers verified models of common optical devices and it is easy to construct new ones by using the design tools of the software. Optical pulse generators, modulators, couplers, delay elements, interferometers, pulse combiners, optical switches, fiber Bragg grating filters, fiber amplifiers, bulk semiconductor amplifiers, and many other optical devices are already available within the software. But optical devices like the optical threshold element and the SOA-MZI switch with backward propagation, which are not included in the software have been designed using signal processing tools or combination of the current models.

\section{Threshold element}

The threshold device can be realized by the combination of two different periodically layered materials with refractive indices of opposite sign [10]. The refractive index of a single layer is the function of the light intensity $I$, linear index coefficient $n_{0}$ and the nonlinear Kerr coefficient $n_{\mathrm{nl}}$.

$n=n_{0}+n_{\mathrm{nl}} I$

The Bragg condition of a linear periodic structure for a medium with intensity dependent refractive indexes could be expressed as [10]:

$\left(n_{01}+n_{\mathrm{n} 11} I\right) d_{1}+\left(n_{02}+n_{\mathrm{n} 12} I\right) d_{2}=\frac{\lambda}{2}$

The Bragg condition is used to determine the spectral position ' $\lambda$ ' of the center of the periodic grating. A stable stop-band, which stays fixed in an intensity dependent medium, could be satisfied if and only the Kerr-coefficients have opposite signs. Required thickness $\left(d_{1}\right.$ and $\left.d_{2}\right)$ for the chosen material set could be determined in terms of linear and nonlinear refractive indexes [10, 11].

The variation of the intensity and refractive index for different values of $I_{\text {in }}$ across the grating period of 1000 has been experimented by Brzozowski [10] with material parameters: $n_{01}=1.5, n_{02}=1.52, n_{\mathrm{n} 11}=0.01$ and $n_{\mathrm{n} 12}=-0.01$. The threshold parameter " $a$ " is defined as $a=\left(n_{01}-n_{02}\right) /$ $\left(\left|n_{\mathrm{n} 11}\right|+\left|n_{\mathrm{n} 12}\right|\right)$ for the given material pairs. Low incident intensity ( $I_{\text {in }}=0.3$ ) is blocked by the grating profile and decays sharply to a negligible intensity level. As the intensity increases closer to $a$ the refractive index variation helps to reflect the incident intensity across the structure (for $I_{\text {in }}=$ 0.65 ). When the incident intensity is equal to $a$ the grating profile will be terminated by the refractive index change and the incident intensity is transmitted completely. As the incident intensity is increased above $a$ (e.g., $I_{\text {in }}=1.07$ ) the grating will be formed again and forces the intensity to be normalized [11]. The approximate piecewise-linear relation between transmitted and incident intensity is as given below [11];

$I_{\text {out }}=\left\{\begin{array}{lll}0 & \text { for } & I_{\text {in }}<a\left(1-1 / 2^{N}\right) \\ a\left[2^{N}\left(I_{\text {in }}-1\right)+1\right] & \text { for } & a\left(1-1 / 2^{N}\right)<I_{\text {in }}<a \\ a & \text { for } & I_{\text {in }}>a\end{array}\right.$

A threshold device will be in transmit state for inputs greater then or equal to threshold value " $a$ ", and the otherwise will be zero as shown in Fig. 2. Furthermore, arranging the proposed structure in series results in increasingly steep transition characteristics.

The fast response of the molecular reorientation makes the Kerr-effect a prime candidate for the use in quick optical gate or optical hard-limiter. In addition, it helps to remove some spikes and noises and normalizes the output. Using the mathematical model in Refs. [10] and [11] the threshold device is designed and simulated in Virtual Photonics simulation software. It is used at the various points of the architecture for different threshold levels.

\section{Pulse extension}

An important step of the algorithm is the extension of the parallel address bits in the time domain. The aim of the pulse extension is to prepare the header information bits for the XOR operation with the routing table pulse train. The routing table is set up as a sequence of pulse trains, which includes all address bits in serial form. In the routing decision procedure, the whole routing table and the each extended address bit have to be XOR'ed together. The uniform Bragg grating filtering method is used to extend the pulse up to the desired duration. The pulse, which is chosen Gaussian, is dispersed in the time domain by adjusting the filter bandwidth and the filter order. Signal amplification is needed as well because of the power lost during the extension step.

A fiber Bragg grating is a periodic perturbation of the refractive index $\left(n_{\text {eff }}\right)$ along the fiber length [5], which in general can be described as [12]:

$\delta_{n_{\mathrm{eff}}}(z)=\dot{\delta}_{n}(z)\left(1+v \cos \left[\frac{2 \pi}{\Lambda} z+\phi(z)\right]\right)$

where $\delta_{n}(z)$ is the "dc" index change spatially averaged over a grating period, $v$ is the fringe visibility of the index change, $\Lambda$ is the nominal period defining filter center frequency and $\phi(z)$ describes the grating chirp. In a uniform filter we used, the refractive index $\delta_{n}(z)$ and $\phi(z)$ are constant. A transfer function of the uniform filters can be calculated analytically $[12,13]$ and is given by: 
Fig. 2 The variation of the intensity for a $\mathrm{N}$-layer grating structure
$T(f)=\frac{\kappa \sinh \sqrt{\kappa^{2}-\xi^{2}}}{\xi \sinh \sqrt{\kappa^{2}-\xi^{2}}+j \sqrt{\kappa^{2}-\xi^{2}} \cosh \sqrt{\kappa^{2}-\xi^{2}}}$

$\kappa$ describes the coupling "strength" between the incident and reflected waves ( $E_{\mathrm{in}}$ and $\left.E_{\text {out }}\right)$ and parameter $\xi$ represents a normalized frequency offset from the center-frequency of the filter. $\kappa$ can be found from the maximum FBG reflectivity $R_{\max }=\left|E_{r} / E_{i}\right|^{2}$ according to $\kappa=\tanh ^{-1}$ $\sqrt{R_{\max }}$ and is defined by the FBG length $L$ and the refractive index perturbation as given by $\kappa=(\pi / \lambda) v \dot{\delta}_{n_{\mathrm{eff}}} L$, where $\lambda$ is the wavelength of the light wave $[12,13]$.

A uniform fiber Bragg grating filter with the transfer function of Equation (5) is used to extend the Gaussian optical pulses with peak pulse power of $1 \mathrm{~mW}$ and $0.1 \mathrm{~ns}$ pulse width. Simulation results include pulse extension and amplification $(\approx 15 \mathrm{~dB})$ of the $0.1 \mathrm{~ns}$ pulses up to the $3.2 \mathrm{~ns}$ [14] as shown in Fig. 3. The simulation parameters are as given below:

$$
\begin{aligned}
& R_{\max }=0.95, \kappa=2.17827, \Delta f_{0}=100 \mathrm{GHz}, \\
& f_{\mathrm{c}}=0, \lambda=1.55 \mu \mathrm{m} ; \Lambda=0.531 \mu \mathrm{m}, v=1, \\
& \delta_{n}=0.0004, L=2.68678 \mathrm{~mm} .
\end{aligned}
$$

The pulse energy efficiency of the pulse extension unit for the ideal Bragg grating extension filtering is calculated as $\approx 91.609 \%$ for 1 -to-32 extension rate [14].

The pulse extension operation could be done by other alternative methods. Optical materials with longer relaxation times might be another solution to the problem. Triggering of this sort of optical materials or semiconductor laser diodes in on-off keying approach may be a solution, too.

\section{All optical XOR operation}

Another critical point of the design is the realization of the SOA assisted MZI switch [9], which will be used as an all-optical XOR gate. The SOA-MZI switch realizes the XOR operation between two pulse streams, routing table pulse train, and parallel address bits. The optical SOA-MZI switch in our study consists of a symmetrical MZI and a bulk SOA with reverse inputs (Fig. 4). Two input signals are fed to the XOR module bidirectionally to interfere at the bulk SOA. The semiconductor amplifier based Mach-Zehnder interferometer posses the practical advantages of low power consumption, low latency, high stability, and integration potential [9]. Although, the simulation is performed for $10 \mathrm{~Gb} / \mathrm{s}$, the SOA-MZI has the potential to operate at $160 \mathrm{~Gb} / \mathrm{s}$ [15]. The parameters of the bulk SOA module of the VPI VirtualPhotonics are optimized through analysis and simulations.

For the switching operation, the RTPT and its inverse RTPT $^{-1}$ enter to the inputs of the two identical bulk SOAs as probe signals. The header bits are functioning as the control signals and fed from the backward propagation input of the bulk SOAs. The parallel header bits are used to drive a clock sequence. When a header clock pulse enters the SOA, it stimulates the recombination of carriers as a response to stimulated amplification. The carrier density decreases sharply on each arrival of a pulse, and it recovers slowly until the next pulse arrives. The fall time is approximately equal to the width of the original pulse. The routing table signal pulse is split into two components and fed into the inputs of the identical bulk SOAs as forward propagating waves. Either the routing table pulse train or its inverse is used as the probe signal at the SOA input. Address header bits are backwardly 


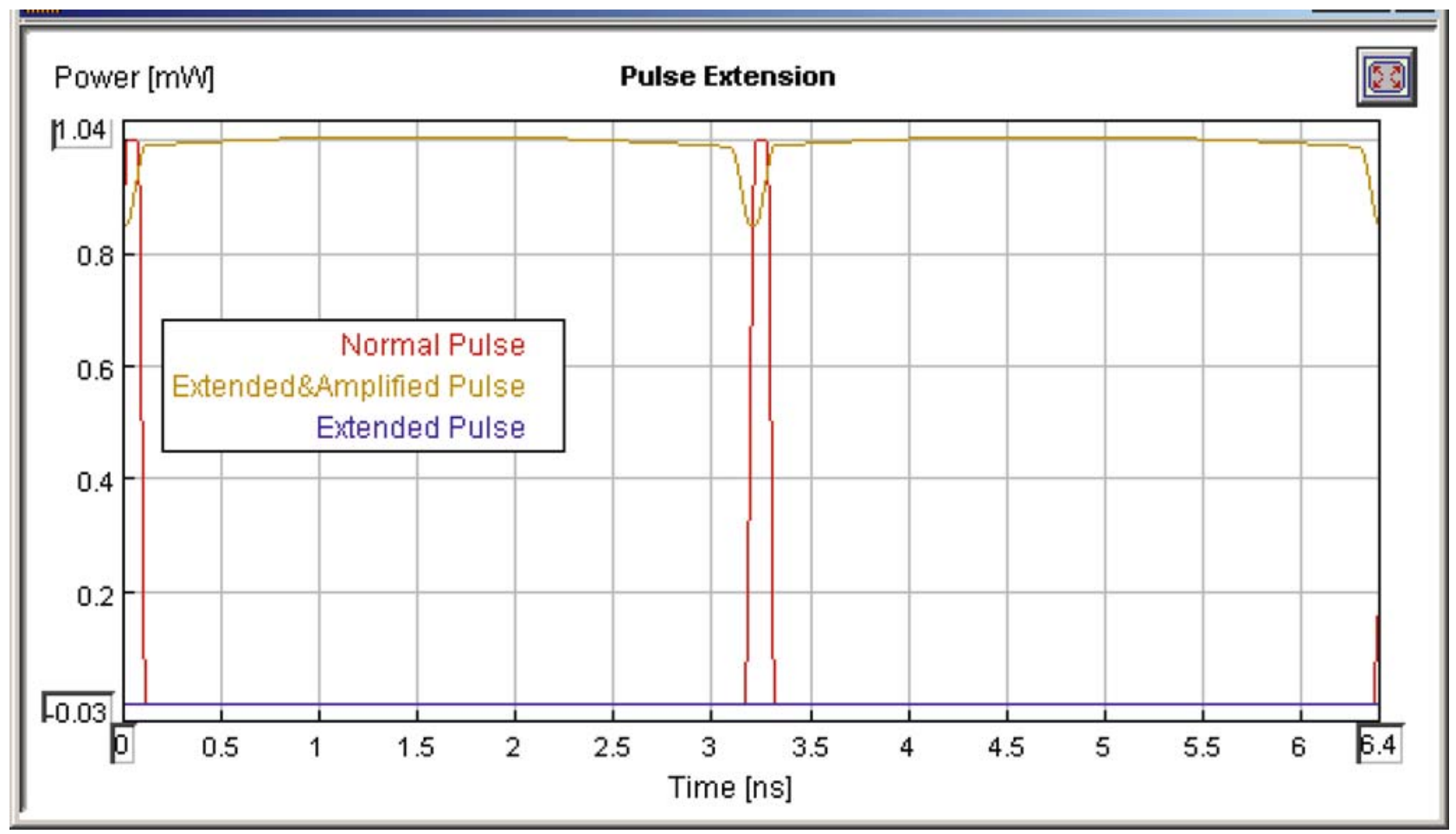

Fig. 3 Extension and amplification of a $0.1 \mathrm{~ns}$ optical pulse

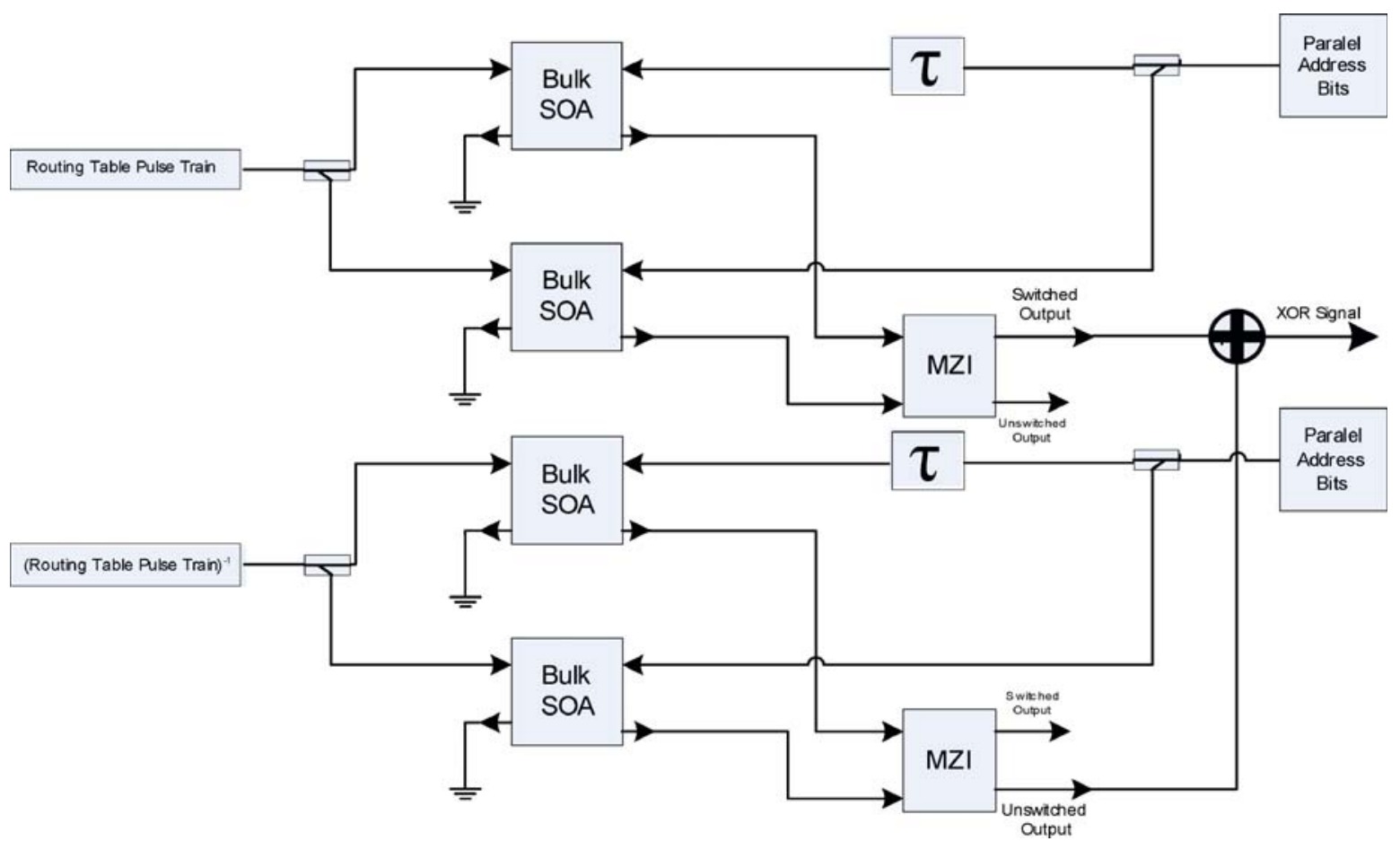

Fig. 4 SOA-MZI switch for optical XOR operation 
Fig. 5 Nonlinear phase shift of SOA

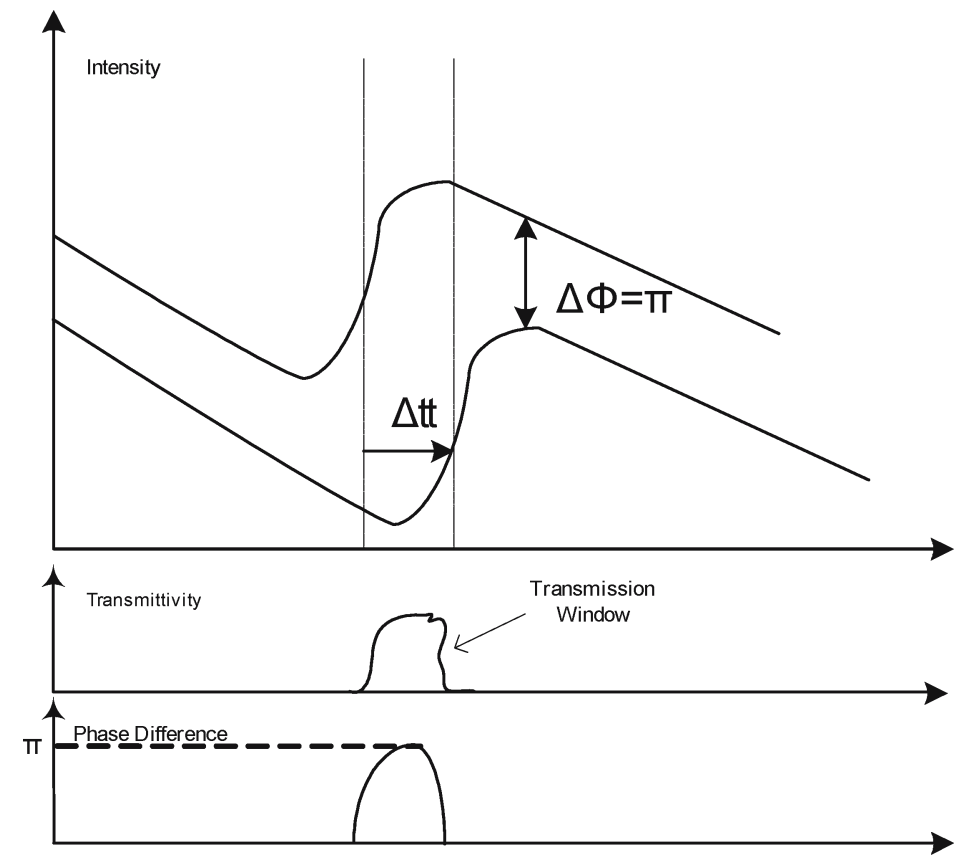

propagated and used to modulate a $\pi$-phase shift inside the SOA. The optical phase shift of each component is adjusted by the nonlinear change in refractive index of the respective SOA, as shown in Fig. 5. This shifting of phase is slightly delayed for one of the pulse trains. This delay time $\Delta t$ is produced by the difference between the times of arrival of the control pulses at the respective SOAs. The two split signal components collide and interfere each other at the interferometer's output. The phase bias between the two components is adjusted such that the phase difference before $t_{0}$ and after $t_{0}+\Delta t$ equals to $\pi$. The rise and fall times of the rectangularlike switching window are determined by the width of the control pulse. The width of the switching window is determined by delay time $\Delta t$ [9]. Each pulse of the clock sequence produces a $\pi\left(180^{\circ}\right)$ phase shift on the probe signal and the MZI opens a near-rectangular switching window and thus gates the routing table pulse train to the switched port. If the header bit is zero, there is no phase shift or switching window on the pulses and there will be no significant signal power at the output.

\section{Simulation results}

The fixed length data packets having three bits of header are generated from On/Off keying modulated Gaussian pulses with $0.1 \mathrm{~ns}$ pulse width and $10 \mathrm{Gbps}$ pulse repetition rate. According to the address information each packet will be sent to either "Route A" or "Route B". In the following example the routing table includes the addresses 000-111-010-100 for "Route A". For every one of these addresses, a routing control bit will be generated to set the optical switch at the output port. Routing table information signals RTPT and its logical inverse $\mathrm{RTPT}^{-1}$ are readily stored in a memory unit and generated by an ultrafast pulse generator.

Table 1 explains the logical operation principle of the routing process and the function of the RTPT, $\mathrm{RTPT}^{-1}$, and synchronization signals. Here, the packet is chosen to carry address " 101 " and because this address is not in the routing table, the routing algorithm should not generate a routing control bit at all. However, the routing table may include the shifted versions of the packets addresses (000111010100, 000111010100) which may result in inter bit matching errors. A synchronization signal whose repetition rate is aligned with the address length has been used to select the right bit sequence. The algorithm selects RTPT for "1" address bits and $\mathrm{RTPT}^{-1}$ for " 0 " address bits to accomplish an XOR operation. If the routing table includes the header address a three optic one's will present consecutively after the XOR operation. Each bit sequence is delayed for one-bit duration from the previous sequence, then summed and passed through an appropriate threshold device to generate the routing control bit. As it can be seen from Table 1, two sets of high-energized pulses (3-pulse energy) have been observed at the pulse combiner, but they should be ignored since they do not overlap with the synchronization pulses.

The simulation results for the "101" addressed packets are shown in Fig. 6. Every bit of the address is separated and converted from serial to parallel (Fig. 6a-d) and they all have been extended in time domain up to the RTPT duration using Fiber Bragg Grating filtering (Fig. 6e-g). A threshold component, as explained in previous section, is used in order to pulse reshaping by reducing the pulse fluctuations and 
Table 1 Logical operation principle of the XOR for "101"

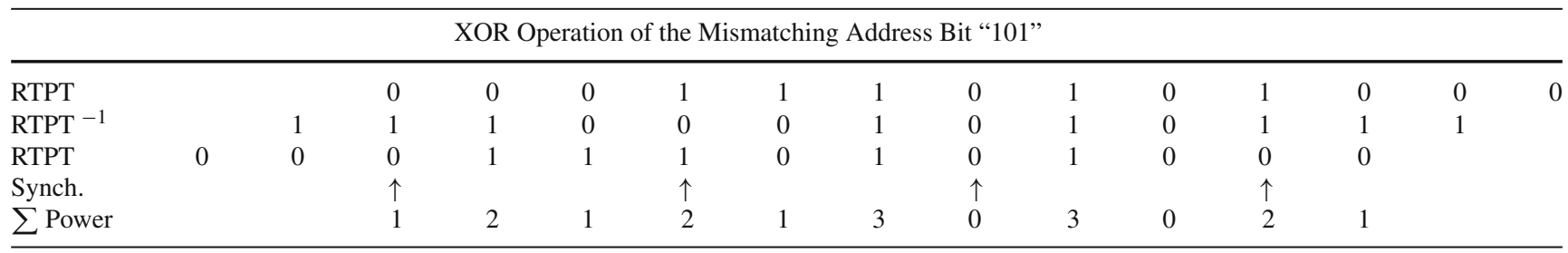

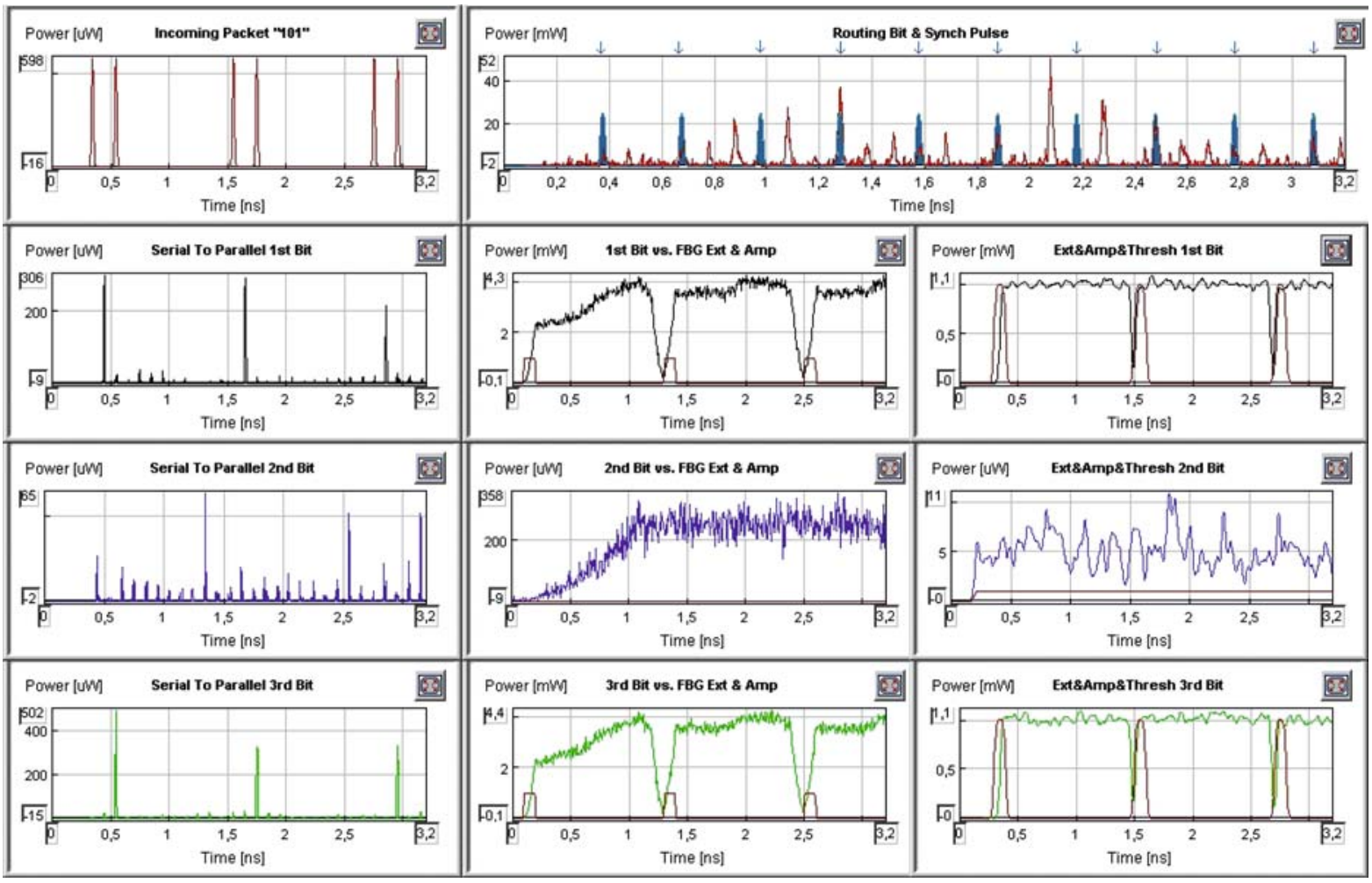

Fig. 6 Simulation results for " 101 "

Table 2 Logical operation principle of the XOR for " 000 "

\begin{tabular}{|c|c|c|c|c|c|c|c|c|c|c|c|c|c|c|c|}
\hline \multicolumn{15}{|c|}{ XOR Operation of the Matching Address Bit " $000 "$} & \\
\hline $\mathrm{RTPT}^{-1}$ & & & 1 & 1 & 1 & 0 & 0 & 0 & 1 & 0 & 1 & 0 & 1 & 1 & 1 \\
\hline $\mathrm{RTPT}^{-1}$ & & 1 & 1 & 1 & 0 & 0 & 0 & 1 & 0 & 1 & 0 & 1 & 1 & 1 & \\
\hline $\mathrm{RTPT}^{-1}$ & 1 & 1 & 1 & 0 & 0 & 0 & 1 & 0 & 1 & 0 & 1 & 1 & 1 & & \\
\hline Synch. & & & $\uparrow$ & & & $\uparrow$ & & & $\uparrow$ & & & $\uparrow$ & & & \\
\hline$\sum$ Power & & & 3 & 2 & 1 & 0 & 1 & 1 & 2 & 1 & 2 & 2 & 3 & & \\
\hline
\end{tabular}

normalizing the pulse peak power to $1 \mathrm{~mW}$ at the SOA-MZI switch input.

For the " 0 " bit the same operation results in low energized noise signals on the order of $\mu \mathrm{W}$ range, in contrast to the $1 \mathrm{~mW}$ for optical " 1 " pulses. Fig. 6k shows the resultant routing control signal and the synchronization pulses (dark pulses marked by arrows) as a result of the algorithm. As seen in the figure the high energized $(\sim 40 \mathrm{~mW})$ pulses do not overlap with the synchronization pulses as expected. The sum of the routing control signal with the synchronization signal should pass through a suitable threshold level for logical AND operation to set the optical switch at the output port. For "101", the routing control bit is not generated and the packet is switched to "Route B" by default.

Table 2 shows the logic table for the "000" addressed packets. Since the "000" address is in the routing table, the routing algorithm should produce a routing control bit to select "Route A". Similarly, the routing control signal 


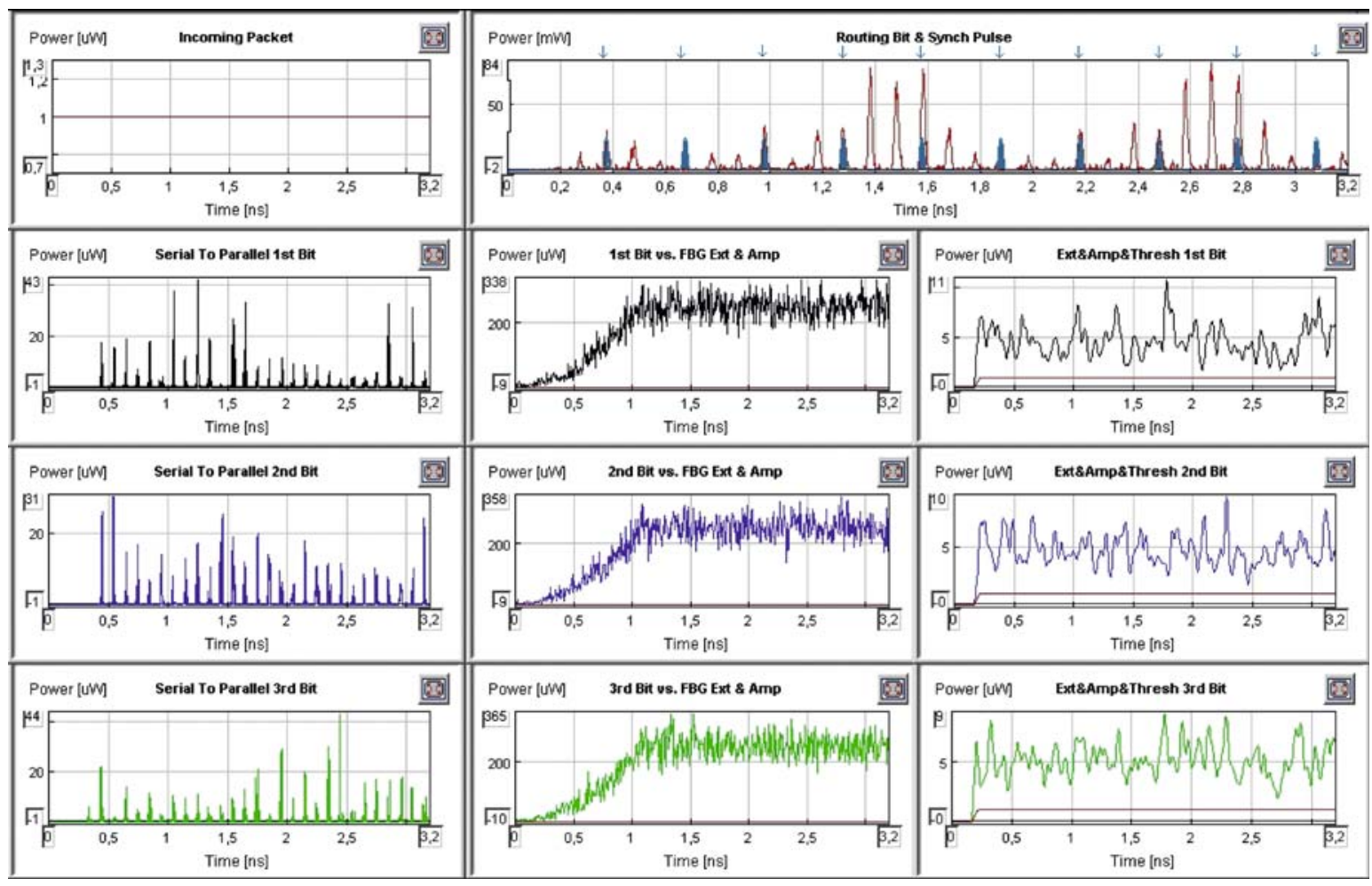

Fig. 7a-k Simulation Results for " 000 "

Table 3 Logical operation principle of the XOR for " 111 " and " 001 "

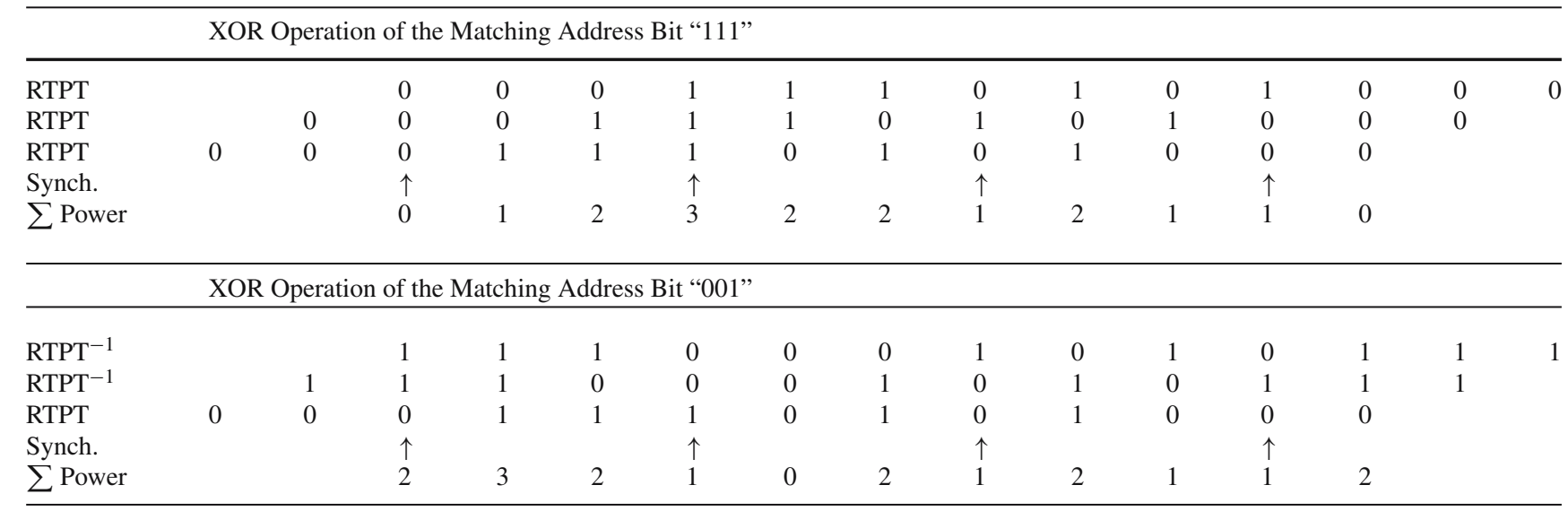

enables the RTPT $^{-1}$ after series of synchronization operations by following the routing procedure.

Figure $7 \mathrm{a}-\mathrm{k}$ shows the simulation results for the " 000 " address. In Fig. 7k, there are three high energized pulses following each other. The synchronization pulses help us to discriminate the right set of the routing control signal. For the packets with "000" addresses a routing control bit should be generated and the packet should be switched to "Route A" as happened in this case.
In the third case, packets with two different addresses are sent to the optical routing node. The first packet is carries the address " 111 " and the second one has address "001". Table 3 shows that the first one should be routed to "Route A" and the second one should be routed to "Route B". The simulation results in Fig. 8 shows that the control bit is generated at $t=0.7 \mathrm{~ns}$ for the first packet and as expected there is, however, no routing control bit produced for the second packet. 


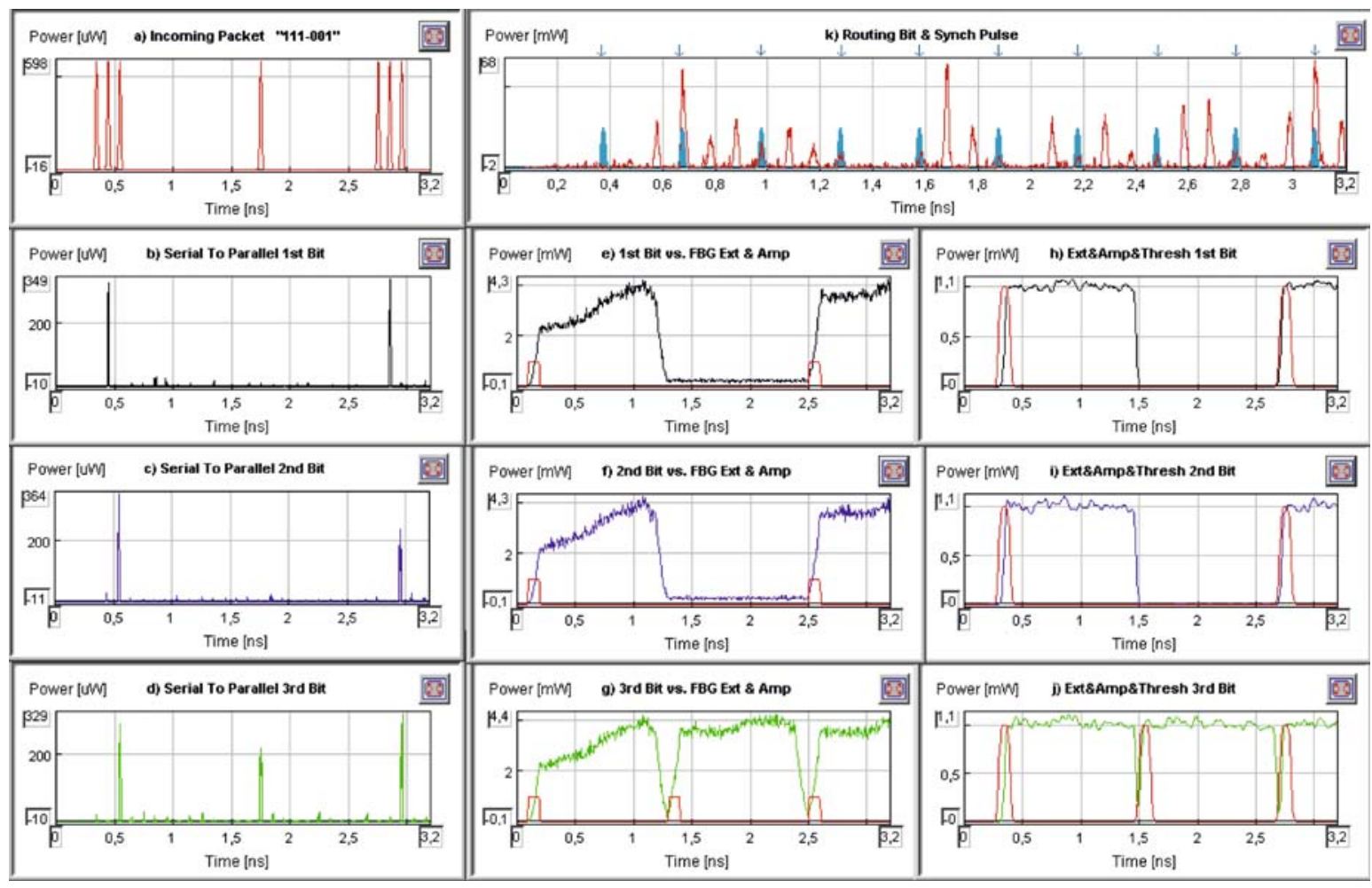

Fig. 8a-k Simulation results for "111" and "001"

\section{Conclusion}

It is important for network designers to reduce the number of protocol layers being used in today's networks, while preserving the functionality. The novel all-optical routing architecture proposed in this work, operates at physical layer of the OSI while providing protocol transparency. The original data packets are routed and sent to the next network node without any data conversion, it remains unchanged. The architecture is very flexible and can be implemented into different kind of networking protocols. It could be efficiently used in autonomous systems like MPLS or in IPv6 backbone routers.

The proposed architecture has been designed using realizable optical signal processing tools. Although the techniques and devices deployed in this architecture are well known for various applications of the optical communication, in this work all these techniques have been improved and exploited to integrate an all-optical routing device.

In the simulation, data packets with $10 \mathrm{Gbps}$ bit rate and 3-bit header have been routed successfully into the desired path, but the architecture has the potential to route at much higher data bit rates since every component used has the characteristics that supports up to $160 \mathrm{Gbps}$. Parallel and serial approaches of the architecture are also possible. The routing table size is another critical factor for most applications.
Routing with address prefixes or a parallel approach can decrease the required routing table size for the architecture.

Synchronization of the data bits is another issue that should be addressed before practical realization of the architecture. Since, in the simulation, the laser source can be excited to fire at well-defined time intervals, which are not the case of the real networks. The delay elements in the simulation are realized by a fixed-length optical cable which may also cause a time jitter problem. However, noise and loss parameters of every component, as VPI allow, are included in the simulation. Many optical signal processing components might suffer from stability problems because of the high data rates. Synchronization and jitter analysis of the architecture is considered as future work of the design.

Next generation networks will most probably rely on optical networking and they require faster architectures at network nodes for optimization of the performance. The proposed all-optical architecture may be an efficient alternative for backbone routers.

\section{References}

1. Desmeu, R.: Implementing Cisco IPv6 Networks (IPv6). Cisco Press (2003)

2. Davies, J.: Understanding IPv6. Microsoft Press (2003) 
3. Tomsu, P., Schmutzer, C.: Next Generation Optical Networks. Prentice Hall (2002)

4. Dinleyici, S., Mocan, B.: Fotonik ağ yapıları için optik sinyal işleme elemanları. Proc. of IEEE SIU 2002, vol. 1. Denizli, pp. 200-205 (2002)

5. Mocan, B., Dinleyici, S.: IEEE SIU 2003 MPLS ağ omurgaları için tüm-optik yönlendirici tasarımı, vol. 1, pp. 79-82 İstanbul, Turkey, (2003)

6. Cotter, D., et al.: Self-routing of $100 \mathrm{Gbit} / \mathrm{s}$ packets using 6 bit 'keyword' address recognition. Electron. Lett. 31(25), 2201-2202 (1995)

7. Boyraz, O.: Time Stretch Optical Header Recognition. LEOS 2003, vol. 2, pp. 543-544 New Jersey, USA, (2003)

8. Takahashi, R., et al.: Ultrafast All-optical Serial-to-parallel Conversion for Optical Header Recognition, vol. 4, pp. 506-507 Amsterdam, Netherland, (2001)

9. Tajima, K., et al.: Ultrafast all-optical signal processing with Symmetric Mach-Zehnder type all-optical switches. Proc. of SPIE 4998, 21-32 (2003)

10. Brzozowski, L.: Optical signal processing using nonlinear distributed feedback structures. IEEE J. Quantum Electron. 36(5), 550555 (2000)

11. Brzozowski, L.: All-optical analog-to-digital converters, hardlimiters, and logic gates. J. Lightwave Technol. 19(1), 114-119 (2001)

12. VPI Virtual Photonics Transmissionmaker 3.1 Photonic Modules Reference Manual. Uniform Fiber Bragg Grating Module, vol. 1, pp. $46-949$

13. Erdoğan, T.: Fiber Grating Spectra, IEEE J. Lightwave Technol. 15(8), 1277-1294 (2001)
14. Mocan, B.: A novel architecture all-optical routing architecture in packet switched computer networks. MS Thesis, İzmir Institute of Technology, İzmir, Turkey (2002)

15. Stubkjaer, K.E.: Semiconductor optical amplifier based all-optical gates for high-speed optical processing. IEEE J. Select. Top. Quantum Electron. 6(6), 1428-1435 (2000)

Bora Mocan received his M.S. degree in Electrical-Electronics Engineering from Izmir Institute of Technology, Turkey in 2002 and B.S. degree in Electrical-Electronics Engineering from Ege University, Turkey, in 1998, respectively. He is currently a Ph.D. student at Dokuz Eylul University, Izmir Turkey, majoring in Electrical-Electronic Engineering. His research interests include optical signal processing, optical Internet, and high performance communication networks.

Mehmet S. Dinleyici received his Ph.D. in Electrical Engineering from Illinois Institute of Technology in 1998. He is currently Assistant Professor in Electrical and Electronics Engineering Department at İzmir Institute of Technology, İzmir Turkey. His current research interests include all-optical communication networks, components for lightwave communication systems, nonlinear optics and various photonics applications for interdisciplinary areas. 